\title{
New Challenges for Clustering in Large Data Base
}

\author{
Archana Tomar \\ Dept. of I.T \\ T.I.T., Bhopal
}

\author{
Deepshikha Patel \\ Dept. of C.S. \\ T.I.T., Bhopal
}

\author{
Nitesh Gupta \\ Dept. of I.T \\ T.I.T., Bhopal
}

\begin{abstract}
Cluster analysis in data mining is a main application of business. This Investigation describes to present NCDBC algorithm that extends expansion seed selection into a DBSCAN algorithm. And the DBSCAN Algorithm describes the density based clustering concept and also describes its hierarchical additional room OPTICS has been planned newly, and one of the mainly triumphant approaches to clustering. Aim of this research work is to move on the hightech clustering; mainly density-based clustering by identifying new challenges for density based clustering and proposing inventive for these challenges. In this work the proposed procedure focuses on decrease the number of seeds points and also reduces the execution time cost of searching neighborhood data. And A hierarchical clustering procedure can be useful to these interesting subspaces in order to calculate a Latitude for north and south cities and also calculate Longitude of different cities.
\end{abstract}

\section{General Terms}

Clustering Algorithms.

\section{Keywords}

Data mining, data clustering, density based clustering, optics algorithm.

\section{INTRODUCTION}

In this work will determine valuable insights from large database this issue has become of growing importance. In this interest has encouraged a rapidly increasing research area, additionally to on a realistic stage with the ease to use of a variety of commercial tools. Unluckily, the general process of this methodology has been restricted with important supposition in Data Mining approaches. In This idea can be made to live in a sole table prevent the exercise of these Data Mining tools inside positive considerable domains, and it will shift the data like a pre processing route. This restriction will provide comparative availability to generate interest in response of Data mining paradigms which will help to generate and then organized data which will provide better result than normal level representation.

The study which is used to produce data by the use of data mining techniques in previous decade, it has been observed that used techniques were extended from well known and approved data mining techniques for the stydu of tabular data. these techniques provide better representation for locating such tabular data. this proposal actually work with previous data mining techniques, to recognize 'ethnicity', the used techniques encouraged to obtain suitable option which will represent the facility to manipulate prepared data.

The proposed work focuses on a practice that approximately emphasis on relational database theory: New Challenges for Clustering in large data base (NCDBCLE).
The relational record theory select data concentrated applications of engineering level. the Relational data base used in this application for data storage and recover. The main reason of the use of relational data base will provide encouraging practical come near on data of Data Mining. This database presumption has a absolute and well-off account of opinion and developments concerning to the well-organized storage and dealing out of prepared data with extensive knowledge of Multi-Relational Data Mining. data mining Concepts which emphasis to provide a model for a data base and normalize that data base will provide the help for NCDBCLE plan. there are many of the Current developments which deal with large volume of data base and use of concentrated processing for data base provide improved application of NCDBCLE in more finer and complicated areas.

In the relational database theory there are various concepts and different customs to encouraged other prepared Data Mining paradigms. NCDBCLE fundamentals various approaches for larger records. the proposed work provide the understandability for relational groundwork. which is helpful for the concept of this proposed work, the proposed work successfully created solution that is not highlighted before in any challenges in this area.

\section{REVIEW}

\subsection{Optimization Problem}

Optimization is the work of obtain the most excellent outcome under certain situation. In propose, structure, and maintenance of at all engineering organization, engineers have to get a lot of technical and administrative, decision at more than a few stages. The final purpose of every part of such decisions is also to reduce the effort necessary or to exploit the preferred advantage. Since the effort necessary or the advantage favored in any realistic condition can be expressed as a function of assured Conclusion variables, optimization can be distinct as the methods of finding the situation that give the highest or lowest value of a job. Optimization, in engineering plan, is a mathematical device which works iteratively on solutions such that objectives similar to rate/presentation /effectiveness etc. are enhanced Optimal problem formulation

The intention of the optimization process is to generate a mathematical model of the optimal design problem, which be able to be solve by an optimization procedure since an optimization procedure admit an optimization problem in a exacting layout, each optimal design problem have to be formulated in that layout. Below shows the basic steps of optimization issues. It consists of different steps:

Select variables for optimization problem

Objective function formulation 


\section{Constraint formulation}

Set up variable bounds

Choose optimization algorithm

Estimate function

Confirm the bounds

\section{Verify Stop Criteria}

The system consisting of generating location's transmission lines and sharing system which give uninterrupted and dependable electrical power is called control system. Power invention optimizations give the information regarding correct loading of generators when there are number of generators having dissimilar unchanging \& variable cost.

\subsection{DBSCAN}

DBSCAN, proposed by Ester et al. in 1996 [17], was the first clustering algorithm to occupy density as a circumstance.

In this part, these work present the DBSCAN are used to locate and find out the clusters, noise in a spatial data base. Generally, and would have to identify the suitable parameters Eps and MinimumPts of every cluster and at least one point from the relevant cluster. After that, we possibly will recover all points with the aim of are density reachable from the specified point by the accurate parameters. In advance there is no simple method to find detail in advance for every clusters of the database. In this algorithm parameters are calculate easily. That's by DBSCAN uses global data for Epspts and MinimumPts. The density parameters clusters are very useful for these global parameters.

\section{DBSCAN Algorithm:}

The DBSCAN algorithm based on Spatial Clustering with Noise [DBSCAN]. this algorithm cluster the high density increased area, and provide resultant clustering shape.

\section{DBSCAN clustering points are:}

1. Epsilon $\varepsilon$ semi diameter of an objective

2. Neighbors in $\varepsilon$ select certain number least points called Core object

3. To an object set $\mathrm{A}$, if object $\mathrm{M}$ is the $\varepsilon$-neighbor of $\mathrm{N}$ and $\mathrm{N}$ is core object, and after that $\mathrm{M}$ can get "direct density reachable" from $\mathrm{N}$.

4. To a $\varepsilon, M$ can get "direct density reachable" from N;A contains Lpts value; if a list of object is $\mathrm{m} 1, \mathrm{~m} 2, \ldots, \mathrm{mn}, \mathrm{m} 1$ $=\mathrm{n}, \mathrm{mn}=\mathrm{m}$, then $\mathrm{m}_{\mathrm{a}}-1$ can get "direct density reachable" from $\mathrm{m}_{\mathrm{a}}, \mathrm{m}_{\mathrm{a}} \in \mathrm{A}, 1 \leq \mathrm{a} \geq \mathrm{n}$.

5. if object $\mathrm{O}(\mathrm{O} \in \mathrm{A})$ exist for $\varepsilon$ and Lpts, $\mathrm{A}$ and $\mathrm{B}$ can obtain "direct density reachable" from $\mathrm{O}, \mathrm{A}$ and $\mathrm{A}$ are density connected.

\subsection{IDBSCAN Algorithm}

IDBSCAN is a density-based data clustering proposal developed by Borah et al. in 2004 [28]. This technique applies a Marked border Object to find out the data point of an expansion seed when searching used for neighborhood to insert in expansion seeds. Assuming that the core point is $\mathrm{P}(\mathrm{O}, \mathrm{O})$, the eight marked border objects may be defined as: A(0, Eps), B(Eps $/ \sqrt{ } 2$, Eps $/ \sqrt{ } 2), C(E p s, 0), D(E p s / \sqrt{ } 2,-$ Eps $/ \sqrt{ }$ $2\}), E(0,-$ Eps $), F(-E p s / \sqrt{ } 2,-$ Eps $/ \sqrt{ } 2)$, G(-Eps, 0), H( -Eps $/ \sqrt{ }$ $2, \mathrm{Eps} / \sqrt{ } 2)$.
If core point indicated with $\mathrm{P}$ and it satisfies the set density condition, after that the Procedure finds inside the neighborhood the nearby point to these eight marked border line objects, and sets these data points like the expansion seeds. Since these seeds may be chosen using multiple marked border objects, the procedure requires just only instance of input is required. The number of seeds point added is below (3di_i), where di represents the dimension of the database.

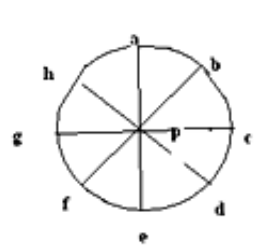

\section{Fig1. Eight marked border object of IDBSCAN}

\subsection{KIDBSCAN Algorithm:}

KIDBSCAN is a density-based clustering technique represent by Tsai and Liu in 2006[18]. In this algorithm searched for marked border objects with IDBSCAN, and found that inputting data in sequence from low density database basis remnant seed searching, consequential in poor expansion results.

To reduce the number of taster instances, this algorithm performs growth by inputting leader points. It has three parameters, leader point, radius and MinimumPts. The implementation steps are as follows.

1. Firstly find the $\mathrm{K}$ numbers of the centroid inside the all database, after that discover the $\mathrm{K}$ data points nearby to this centroid and describe them as leader points, because K-means can discover these leader points quickly.

2. Shift the $\mathrm{K}$ leader points to the extremely front of the database.

3. Perform the IDBSCAN algorithm. Investigational results show that KIDBSCAN performs data clustering quickly.

\section{TOOL FOR COMPARISON}

\subsection{Inspiration}

The meaning of comparison in application areas for example searching the location of many cities in the world, marketing, computer aided engineering, molecular biology, medical imaging and purchasing support etc. Generally, the most important work of the finding related shapes in 2-dimention and 3-Dimention. Example for new applications that want to recovery of related 3-dimensional items consist of databases for medical imaging, computer aided design and molecular biology.

In this research work, show the original application ranges of density based hierarchical clustering which led to the expansion of comparison tool called BOSS OPTICS algorithm for Similarity Search. The main idea of BOSS is to provide a brows able hierarchy of clusters each represented by one or more significant objects.

- Compare to another application OPTICS is callous to its two key frameworks, epsilon and Minimumpts. The key condition at present has to exist in plentiful to defer good results. -This application is also a hierarchical clustering. Which returns plentiful information of the group structure than a modus that estimate a smooth separate of the data . 
- In the large data base this modus is associable and scalable. The accomplishment of this algorithm can be very much made better through stepping-up the extent queries, utilizing correct spatial indication frame[].

- The eventuality of this modus is a cluster ordering. With obtainable scheme cluster orderings can be vision abundant bounteous clear than dendrograms particularly for abundant data sets.

\subsubsection{Visual Data Mining}

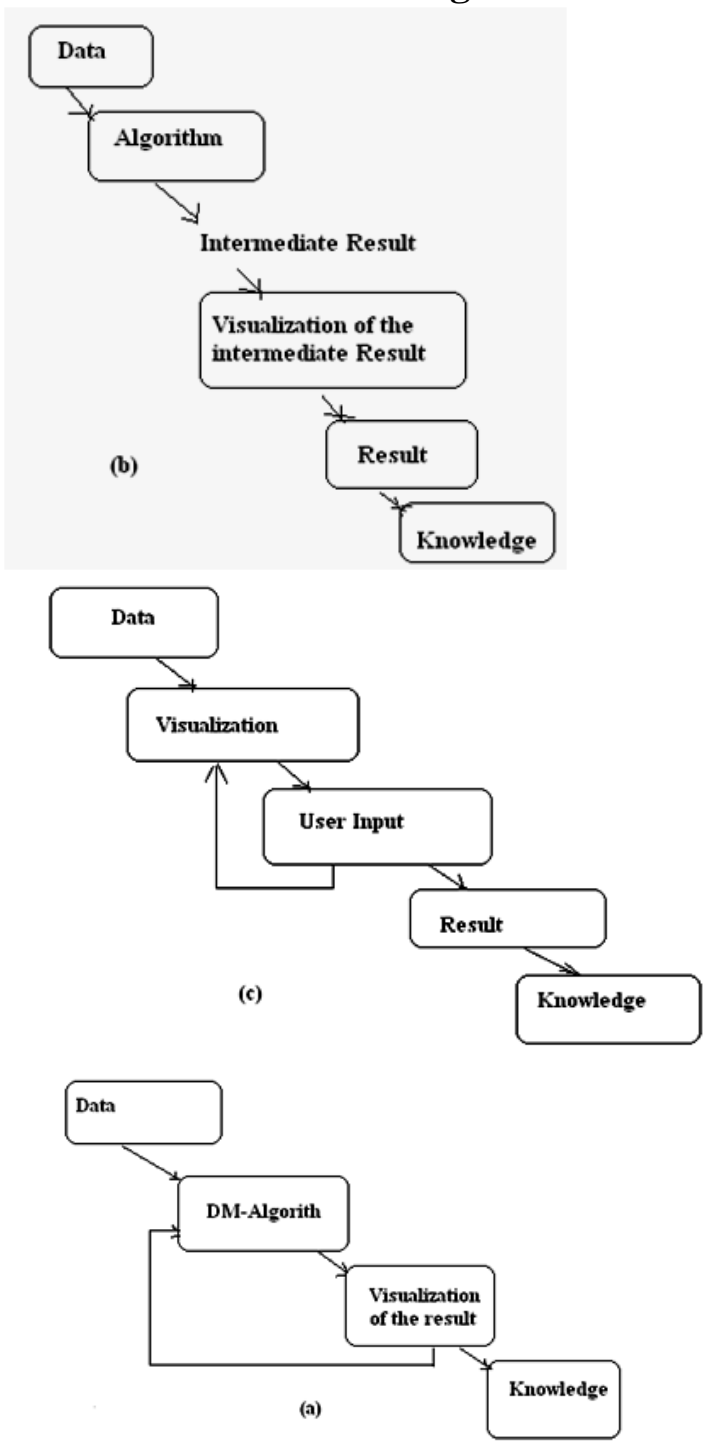

Fig 2: multiple approaches to visual data mining

\subsubsection{Similarity Search}

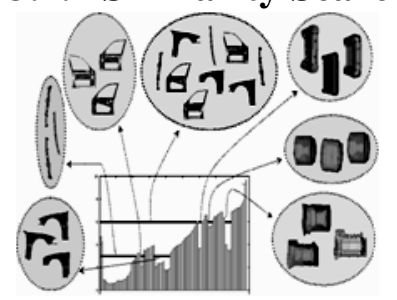

Fig 3: interactive data browsing tool

\subsection{Requires Enhancement}

The BOSS prototype uses the information of a reachability plot generated by OPTICS to support the three sketched applications by visualizing the hierarchical clustering structure, generating a hierarchy of clusters, and re- vealing representative objects of each cluster. However, some steps in the pipeline from the raw data to the interactive browsing remain unsolved so far. In particular, to enable browsing a hierarchy of cluster representatives extracted from a reachability plot, several additions and enhancements to the hierarchical clustering algorithm OPTICS are necessary. In the following, this work of two requirements that were needed to be addressed during the development of BOSS.

\section{IMPLEMENTATION OF TECHNIQUE}

1.Initialization:- Initialize all parameters, and define a new Cluster ID. and will set generator counter is set to $\mathrm{G}=1$.

2.valuation:- Begin scanning all data points within the entire database. For data points belonging to the Clust_ID Of those unclassified data, implement the Expand Cluster processing procedure. The database is the set of data points; the Point represents the core point; the Clust_ID denotes the current cluster ID;e indicates the radius, and MinPts represents the minimum number of included points.

3.Replacement:- If the data point returned by the expansion procedure function is a Noise data point, then go directly to Step2, until the Datasets database has been fully scanned. If an expansion data point is returned, then update the new Cluster ID, and alter the index array of unclassified data, and then go to Step 2.

4. Termination: - End the algorithm when all data points have been processed.

\subsection{Procedure for solving Problem using clustering technique}

The execution process for the increase Cluster processing steps are as follows.

1. Search for neighborhood data within the range of radius e in the unclassified cluster index. If the number of neighborhood data is less than MinPt, then leave the procedure, and return the core point as the noise data point. Otherwise, go to Step 2

2. Set center point as the current Clust_ID.

3. But the seed point is null then stop the expansion processing method, or else go to next Step.

4. And then Search for the boundary spot within neighborhood records, then add in the expansion seeds.

5. Place all unclassified and neighborhood data points that are noise data as the current Clust_ID.

6. Extract the first seed from the expansion seeds; define it as the core point, and then delete it.

7. in the unclassified data index, search for neighborhood data within the range of radius e of the core point. If the number of neighborhood data is greater than Min Pts, then go to Step 3. 


\section{EXPERIMENTAL RESULTS}

The Clustering algorithm and finding the location of various cities with latitude and longitude in world was implemented in the Python and PHP language running in windows XP. According to the Results the proposed NCDBCLE outperforms the related density based clustering approaches involving DBSCAN, IDBSCAN and KIDBSCAN. This work applies the NCDBCLE to conduct clustering application. The sunset time and sunrise time database obtaining data from the proposed application.

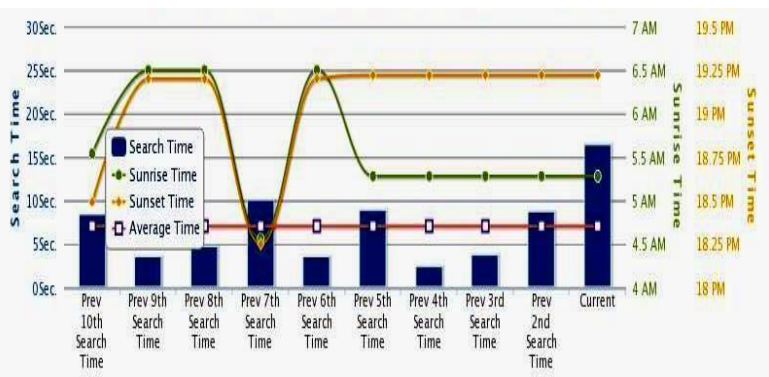

Fig 4: Last 10 Execution Time, Sunset Time, Sunrise Time and Average Time

And the result of the data clusters using NCDBCLE approach where epsilon and MinimumPts is $15 \mathrm{k}$ and 2.

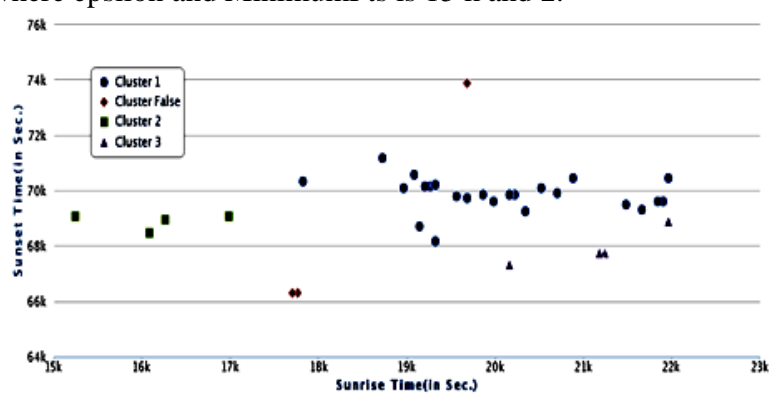

Fig 5: Clustering on Sunrise and Sunset Time by Proposed application

\section{CONCLUSION}

This dissertation introduces a new challenge cluster optimization approach for the problem related to data mining. This algorithm provides efficient working of data clustering with expansion of seed point efficiently. The working principal of this algorithm reduces eight Marked Boundary Objects, which resultant into coverage growth. The planned process accomplish brilliantly for arbitrary shapes and if the no of data points increases the computational time does not increase for large data sets there is no limitation by memory.

\section{REFERENCES}

[1] U. Fayyad, P. Smyth, and G.P. Shapiro. 1996. Knowledge Discovery and Data Mining.

[2] Heikki Mannila, P. Smyth and David Hand. 2001. Principles of Data Mining.

[3] Arno. jan knobbe. 2004. Multi Relational Data mining.

[4] Ke-bing Zang. 2007. Visual Clustering analysis in data mining.

[5] Diane J. Cook and Lawrence B. Holder. 2000. GraphBased Data Mining, Intelligent Systems \& their Applications.

[6] Takashi Matsuda, Hiroshi Motoda and Takashi washio. 2002. Graph-bases induction and it's allpication.

[7] Handrik Blockeel. 1998. Top-Down Induction of First Order Logical Decision Trees.

[8] Luc De Raedt and Handrik Blockeel. 1998. Top-down induction of first order logical decision trees.

[9] Demoen B, Handrik Blockeel. and Jacobs N. 1999. Scaling up inductive logic Programming learning from interpretations and Data Mining and knowledge Discovery.

[10] Dehaspe, L. 1998. Frequent Pattern Discovery in FirstOrder Logic.

[11] Džeroski S. 1996. Inductive Logic Programming and Knowledge Discovery in Databases.

[12] Džeroski S. and Lavrač N. An Introduction to Inductive Logic Programming.

[13] N. Lavrac and Saso D. 1994. Inductive Logic Programming: Techniques and Applications.

[14] Tsai C.F. and Liu C. w. 2006. A New Efficient Data Clustering Algorithm for Data Mining in Large Databases.

[15] Tsai C.F. and Yen C.C. 2007. A New Effective and Efficient Hybrid Clustering Technique for Large Databases.

[16] Saso D. 2001. From Inductive Logic programming to Relational Data Mining.

[17] Progol Muggleton and S. Inverse entailment. 1995. New Generation Computing.

[18] De Raedt L. 1996. Advances in Inductive Logic Programming.

[19] Abe K., Kawasoe S., Asai T., Arimura H. and Arikawa S. 2002. Optimized Substructure Discovery for Semi Structured Data.

[20] Kawasoe S., Abe K., Arikawa S. and Arimura H. 2000. Optimized Substructure Discovery for Semi-Structured Data.

[21] Klemettinen D. and M. Braga. 2002. Mining Association Rules from XML Data.

[22] Shoudai T., Ueda H, Uchida T. and Takahashi K. 2001. Discovery of frequent tree structured patterns in semi structured web documents.

[23] Sander J., Kriegel H. and Ester M.. 1996. A DensityBased Algorithm for Discovering Clusters in Large Spatial Databases with Noise.

[24] Xu, R. and Wunsch, D. 2005. Survey of Clustering Algorithm, mEE Transactions on Neural Networks. 